\title{
Modification in Gravitational Waves Production Triggered by Spontaneous Lorentz Violation
}

\author{
Kevin M. Amarilo*, Mapse Barroso F. Filho and Roberto V. Maluf \\ Universidade Federal do CearÃa, Brazil \\ E-mail: kevinefisica.ufc.br, mapseefisica.ufc.br, \\ r.v.malufefisica.ufc.br
}

In this work, we search how the production of gravitational waves is corrected by a spontaneous Lorentz violation triggered by the vacuum expectation value (VEV) of the bumblebee vector field $\left(b^{\mu}\right)$. We consider the modified graviton equations of motion by the dynamics of the bumblebee field. In this formulation the graviton is still non-massive and has two degrees of freedom, but does not has a gauge transformation and the dispersion relation changes from $p^{2}=0$ to $p^{2}+$ $\xi\left(b^{\mu} p_{\mu}\right)^{2}=0$. Then, we solve these equations considering a current that acts as a source of the radiation. In this case, we apply the method of the Green's function to find a modified quadrupole formula for the perturbation.

International Conference on Black Holes as Cosmic Batteries: UHECRs and Multimessenger AstronomyBHCB2018

12-15 September, 2018

Foz du Iguazu, Brasil

\footnotetext{
* Speaker.
} 


\section{Introduction}

The idea that the Lorentz symmetry may not hold in greater energies scales began in the context of the string theory [1]. Since then, the investigation of the Lorentz Symmetry Breaking (LSB) was developed into the effective field theory known as Standard Model Extension (SME), in which the usual fields of the SM and GR are coupled with fixed background fields that trigger the Lorentz and CPT Breaking.

Among the fields that break the Lorentz Symmetry, the bumblebee field $\left(B_{\mu}\right)$ is one relatively simple. The symmetry is spontaneously broken by the dynamics of $B_{\mu}$ that acquires a nonzero vacuum expectation value (VEV) $[2,3]$. This model was first considered with the LSB being triggered by a Higgs-like potential $V\left(B^{\mu}\right)=\lambda\left(B^{\mu} B_{\mu} \mp b^{2}\right)^{2} / 2$.

In this work, we analyze the repercussion of the LSB triggered by the bumblebee model in the production of Gravitational Waves (GW) scenario. We start modifying the free wave equation for the graviton. Then, a current $J_{\mu v}$ is added to the model and a Green's function is derived for the timelike $b^{\mu}\left(b^{0}, 0\right)$ and spacelike $b^{\mu}=(0, \mathbf{b})$ configurations for the bumblebee VEV. With these results, the Green's Function for the modified equation will be compared with the usual one, showing the modifications in the theory.

\section{Modifying the Graviton's Wave Equation}

The extension of the gravitational sector including the Lorentz-violating terms is given by the action

$$
S=S_{E H}+S_{L V}+S_{\text {matter }} .
$$

The first term refers to the usual Einstein-Hilbert action

$$
S_{E H}=\int d^{4} x \sqrt{-g} \frac{2}{\kappa^{2}}(R-2 \Lambda),
$$

where $\mathrm{R}$ is the curvature scalar and $\Lambda$ is the cosmological constant, which will not be considered in this analysis.

The $S_{L V}$ term consists in the coupling between the Bumblebee field and the curvature of spacetime. The leading terms are

$$
S_{L V}=\int d^{4} x \sqrt{-g} \frac{2}{\kappa^{2}}\left(u R+s^{\mu v} R_{\mu v}+t^{\alpha \beta \mu v} R_{\alpha \beta \mu v}\right),
$$

where $u, s^{\mu v}$ and $t^{\alpha \beta \mu v}$ are dynamical fields with zero mass dimension.

Finally, $S_{M}$ takes account the matter-gravity couplings, which in principle should include all fields of the standard mode model as well as the possible interactions with the coefficients $\mathrm{u}, s^{\mu v}$ and $t^{\alpha \beta \mu v}$.

The gravity-bumblebee coupling can be represented by the action (2.3) defining

$$
u=\frac{1}{4} \xi B^{\alpha} B_{\alpha}, \quad \xi\left(B^{\mu} B^{v}-\frac{1}{4} g^{\mu v} B^{\alpha} B_{\alpha}\right), \quad t^{\alpha \beta \mu v}=0 .
$$

The dynamics of $B^{\mu}$ is dictated by the action

$$
S_{B}=\int d^{4} x \sqrt{-g}\left[-\frac{1}{4} B^{\mu v} B_{\mu v}+\frac{2 \xi}{\kappa^{2}} B^{\mu} B^{v} R_{\mu v}-V\left(B^{\mu} B_{\mu} \mp b^{2}\right)\right],
$$


where we introduced the field strength $B_{\mu v}$

$$
B_{\mu v}=\partial_{\mu} B_{v}-\partial_{v} B_{\mu}
$$

in analogy with the electromagnetic field tensor $F_{\mu \nu}$, in fact, the bumblebee models are not used only as a toy model to investigate the excitation originated from the LSB mechanism, but also as a alternative to $\mathrm{U}(1)$ gauge theory for the photon. In this theory, the photon appears not as a fundamental particle, but as a Nambu-Goldstone mode due to spontaneous Lorentz violation [4].

The potential, defined as

$$
V=\frac{\lambda}{2}\left(B^{\mu} B_{\mu} \mp b^{2}\right)^{2},
$$

is responsible for triggering the spontaneous breakdown of diffeomorphism and Lorentz symmetry. Here $b^{2}$ is a positive constant that stands for the nonzero expectation value of this field.

In the pursue of the influence of the gravity-bumblebee coupling on the graviton, we must assess the linearized version [7]. Therefore, we split the dynamic fields into the vacuum expectation values and the nearby quantum fluctuations:

$$
\begin{aligned}
g_{\mu v} & =\eta_{\mu v}+\kappa h_{\mu v}, \\
B_{\mu} & =b_{\mu}+\tilde{B}_{\mu},
\end{aligned}
$$

where $h_{\mu v}$ and $B_{\mu}$ represent small perturbations around the Minkowski background and a constant vacuum value $b^{\mu}$, respectively.

Following the procedure described in Ref. [7], the solution for the linearized bumblebee equation of motion in the momentum space is

$$
\tilde{B}^{\mu}=\frac{\kappa p^{\mu} b_{\alpha} b_{\beta} h^{\alpha \beta}}{2(b \cdot p)}+\frac{2 \sigma b_{\alpha} R^{\alpha \mu}}{p^{2}}-\frac{2 \sigma p^{\mu} b_{\alpha} b_{\beta} R^{\alpha \beta}}{p^{2}(b \cdot p)}+\frac{\sigma p^{\mu} R}{4 \lambda(b \cdot p)}-\frac{\sigma b^{\mu} R}{p^{2}}+\frac{\sigma p^{\mu} b^{2} R}{p^{2}(b \cdot p)},
$$

with $p^{\mu}=\left(p^{0}, \mathbf{p}\right), \sigma=\left(2 \xi / \kappa^{2}\right)$, while $R_{\mu \nu}$ and $R$ are taken in their linearized form. This solution can be inserted in the Lagrangian (2.3) as specified in [5] leading to the following effective Lagrangian

$$
\begin{aligned}
\mathscr{L}_{L V} & =\xi\left[p^{2} b_{\mu} b_{v} h^{\mu v} h_{\alpha}^{\alpha}+\frac{1}{2}(b \cdot p)^{2}\left(h_{\alpha}^{\alpha}\right)^{2}\right. \\
& \left.-\frac{1}{2}(b \cdot p)^{2} h^{\mu v} h_{\mu v}+p^{2} b_{\mu} b_{v} h^{\mu \alpha} h_{\alpha}^{v}-\left(b_{\mu} b_{v} p_{\alpha} p_{\beta}+b_{(\mu} p_{v)} b_{(\alpha} p_{\beta}\right) h^{\mu v} h^{\alpha \beta}\right] \\
& +\frac{4 \xi^{2}}{\kappa^{2}}\left[\left(-2 p^{2} b_{\mu} b_{v}-2 b^{2} p_{\mu} p_{v}+4 b \cdot p b_{(\mu} p_{v)}-\frac{p^{2} p_{\mu} p_{v}}{4 \lambda}\right) h^{\mu v} h_{\alpha}^{\alpha}\right. \\
& \left.+\left(2 b_{\mu} b_{v} p_{\alpha} p_{\beta}-b_{(\mu} p_{v)} b_{(\alpha} p_{\beta}\right)+\frac{b^{2} p_{\mu} p_{v} p_{\alpha} p_{\beta}}{p^{2}}-\frac{2(b \cdot p) p_{\mu} p_{v} b_{(\alpha} p_{\beta)}}{p^{2}}+\frac{p_{\mu} p_{v} p_{\alpha} p_{\beta}}{4 \lambda}\right) h^{\mu v} h^{\alpha \beta} \\
& \left.+\left(b^{2} p^{2}-(b \cdot p)^{2}+\frac{p^{4}}{4 \lambda}\right)\left(h_{\alpha}^{\alpha}\right)^{2}+\left(p^{2} b_{\mu} b_{v}-2(b \cdot p) b_{(\mu} p_{v)}+\frac{(b \cdot p)^{2} p_{\mu} p_{v}}{p^{2}}\right) h^{\mu \lambda} h_{\lambda}^{v}\right]+\mathscr{O}\left(h^{3}\right),
\end{aligned}
$$


where $h=h_{\alpha}^{\alpha}$, it is worth noting that this Lagrangian is not invariant under the usual gauge transformation of the metric perturbation $\left(h_{\mu \nu} \rightarrow h_{\mu \nu}+i p_{\mu} \zeta_{v}+i p_{\nu} \zeta_{\mu}\right)$

Proceeding with the analysis, we must add the Lorentz-violating terms of Eq. (2.10) to the bilinear terms of the Einstein-Hilbert Lagrangian

$$
p_{\mu} p_{\alpha} h^{\mu v} h_{v}^{\alpha}-p_{\mu} p_{v} h^{\mu v} h+\frac{1}{2} p^{2} h_{\mu}^{\mu} h-\frac{1}{2} p^{2} h^{\mu v} h_{\mu v},
$$

forming the kinetic Lagrangian

$$
\mathscr{L}_{\text {kin }}=\mathscr{L}_{E H}+\mathscr{L}_{L V}
$$

Next, we must evaluate the Feynman propagator of the graviton using this Lagrangian, therefore first we rewrite the $\mathscr{L}_{\text {kin }}$ into the bilinear form

$$
\mathscr{L}_{k i n}=-\frac{1}{2} h^{\mu v} \hat{\mathscr{O}}_{\mu v, \alpha \beta} h^{\alpha \beta},
$$

where this operator is symmetric in the indices $(\mu v),(\alpha \beta)$ (the same way as $h^{\mu v}$ and $h^{\alpha \beta}$ ) and under the interchange of the pairs $(\mu v)$ and $(\alpha \beta)$. In this notation, the graviton propagator defined as

$$
\left\langle 0\left|T\left[h_{\mu v}(x) h_{\alpha \beta}(y)\right]\right| 0\right\rangle=D_{\mu v, \alpha \beta}(x-y),
$$

where $D_{\mu v, \alpha \beta}(x-y)$ is the operator that satisfies the Feynman's propagator equation, given as

$$
\hat{\mathscr{O}}_{\lambda \sigma}^{\mu \nu,} D^{\lambda \sigma, \alpha \beta}(x-y)=i \mathscr{I}^{\mu v, \alpha \beta} \delta^{(4)}(x-y)
$$

where $\mathscr{I}^{\mu v, \alpha \beta}=\frac{1}{2}\left(\eta^{\mu \alpha} \eta^{\nu \beta}+\eta^{\mu \beta} \eta^{v \alpha}\right)$ is the identity operator. From this point, the problem of determining the Feynman propagator is reduced to the inversion of the $\hat{\mathscr{O}}$ operator. One method for obtaining the inverse of rank two tensors is based on the Barnes-Rivers spin projection operators. The process, which is considerable tedious, and the propagator is found in Ref. [6]. The propagator has two poles, that represents two dispersion relations for the graviton,

$$
p^{2}+\xi(b \cdot p)^{2}=0 ; \quad(b \cdot p)^{2}-b^{2} p^{2}=0 .
$$

The first mode has no problems concerning the stability and causality, but the second, even though preserves the causality, is non-unitary and spoils the physical consistency.

The equation of motion obtained from the effective Lagrangian (2.12) is

$$
\hat{\mathscr{O}}_{\mu v, \alpha \beta} h^{\alpha \beta}=0 \text {. }
$$

The Eq. (2.17) is then saturated with $p^{\mu} p^{v}, b^{\mu} b^{v}, p^{(\mu} b^{v)}$ and the Minkowski metric, revealing the following constraints

$$
p_{\mu} p_{v} h^{\mu v}=0 ; \quad b_{\mu} b_{v} h^{\mu v}=0 ; \quad p_{(\mu} b_{v)} h^{\mu v}=0 ; \quad h=0 .
$$

Even more constraints can be found saturating (2.17) with $p^{\mu}$ and $b^{\mu}$ alone, which leads to

$$
p_{\mu} h_{v}^{\mu}=0 ; \quad b_{\mu} h_{v}^{\mu}=0 .
$$


The Eqs. (2.18)-(2.19) consist in a set of 12 constraints that can reduce the 14 degrees of freedom of the theory (ten of the graviton and four of the bumblebee field). Therefore, we are left with two physical degrees of freedom, which is the same number of the usual Einstein-Hilbert's graviton.

We apply the Eqs. (2.18)-(2.19) to the Eq. (2.17), and it simplifies to

$$
\left[p^{2}+\xi(b \cdot p)^{2}\right] h_{\mu v}=0
$$

that is the correct energy-momentum dispersion relation associated with the physical pole determined in the Eqs. (2.16). Therefore, we can conclude that the spontaneous Lorentz violation triggered by the bumblebee field modified the Einstein-Hilbert's dispersion relation as $p^{2}=0 \rightarrow$ $p^{2}+\xi(b \cdot p)^{2}=0$. Moreover, the non-minimal coupling $B^{\mu} B^{v} R_{\mu \nu}$ has not produced massive modes for the graviton.

\section{The Production of Gravitational Waves in the Presence of the Bumblebee Field}

For the analysis of the production of GW we add a current $J_{\mu \nu}$ that acts as a source to the dispersion relation in Eq. (2.20) resulting in the equation

$$
\left[p^{2}+\xi(b \cdot p)^{2}\right] h_{\mu v}=J_{\mu v}
$$

The method of the Green's function is commonly used to solve this kind of differential equation. The Green's function must satisfy

$$
\hat{\mathscr{O}} G(x-y)=\delta^{(4)}(x-y),
$$

here $\hat{\mathscr{O}}=\partial^{2}-\xi\left(b^{\mu} \partial_{\mu}\right)^{2}$, which is the dispersion relation in the configuration space. Defining $G(x-y), h_{\mu v}$ is determined by the convolution integration

$$
h_{\mu v}(x)=\int d^{4} y G(x-y) J_{\mu v}(y) .
$$

In the usual Einstein-Hilbert linearized theory, $G_{E H}(x-y)$ is the Retarded Green's function

$$
G_{R}(x-y)=\frac{1}{4 \pi r} \delta[\tau-r] \Theta(\tau),
$$

here it was considered that the four-vectors are divided into a temporal and a spatial part $\left(x^{\mu}=\right.$ $\left.\left(x^{0}, \mathbf{x}\right)\right)$ in this scenario, $\tau=x^{0}-y^{0}, r=|\mathbf{x}-\mathbf{y}|$ and $\Theta(x)$ is the Heaviside step function. The convolution leads to the quadrupole formula of $h_{\mu v}$ considering a non-relativistic, isolated and far-away object.

For the modified graviton's equation, we can represent the Green's function in the momentum space, solving the Eq. (3.2) we get

$$
\tilde{G}(p)=\frac{1}{p^{2}+\xi(b \cdot p)^{2}},
$$


then to get $G$ in the configuration space, we may use the inverse Fourier Transform. The inversion is made for two particular cases, the first is considering that $b^{\mu}$ has a timelike configuration $b^{\mu}=$ $\left(b^{0}, \mathbf{0}\right)$, in this case the Green's function resumes to

$$
\tilde{G}(p)=\frac{1}{\left[1+\xi\left(b^{0}\right)^{2}\right] p_{0}^{2}-|\mathbf{p}|},
$$

and the inversion results to

$$
G(x-y)=\frac{1}{4 \pi \sqrt{1+\xi\left(b^{0}\right)^{2}} r} \delta\left[r-\frac{\tau}{\sqrt{1+\xi\left(b^{0}\right)^{2}}}\right],
$$

which differs from the Eq. (3.4) only by the factors $\sqrt{1+\xi\left(b^{0}\right)^{2}}$. Considering that $b^{0}$ must be small in comparison to the components of the perturbation, this factor will be slightly greater than the unit.

The second case is a spacelike configuration $b^{\mu}=(0, \mathbf{b})$, which will reduce $\tilde{G}(p)$ to

$$
\tilde{G}(p)=\frac{1}{p_{0}^{2}-\left(1-\xi|\mathbf{b}|^{2} \cos ^{2} \Psi\right)|\mathbf{p}|},
$$

here, $\Psi$ is the angle between $\mathbf{p}$ and $\mathbf{b}$, therefore $\cos ^{2} \Psi=\cos (\theta) \cos \left(\theta_{b}\right)+\sin (\theta) \sin \left(\theta_{b}\right) \cos \left(\phi_{b}-\right.$ $\phi)$, with $(\theta, \phi)$ and $\left(\theta_{b}, \phi_{b}\right)$ the angular coordinates of the trivectors $\mathbf{p}$ and $\mathbf{b}$, respectively.

The inverse Fourier transform of Eq. (3.8) expanded to the second order of $|\mathbf{b}|$ is

$$
G(x-y)=G_{R}(x-y)-G_{2}(x-y),
$$

the second term is defined as

$$
\begin{aligned}
G_{2}(x-y)= & \frac{\xi b^{2}}{8 \pi r^{3}} \Theta(\tau) \times \\
& \times\left\{(b \cdot r)^{2}\left[\left(\frac{\tau}{r}-1\right) \delta(\tau-r)+\tau \delta^{\prime}(\tau-r)\right]+\tau r b^{2} \cos \left(2 \theta_{b}\right) \delta(\tau-r)\right\} .
\end{aligned}
$$

This term of the Green's function highlights an interesting property of the bumblebee models, the existence of an anisotropy in the solutions [8]. The vector $\mathbf{b}$ selects a preferential direction for the propagation of the wave.

The solution for the perturbation, considering that the source has a slow motion, is the wellknown quadrupole formula

$$
h_{i j}(t, \mathbf{r})=\frac{2 G}{r} \frac{d^{2} I_{i j}}{d t^{2}}\left(t_{r}\right),
$$

where $t_{r}=t-r$ is the retarded time and $I_{i j}$ is the quadrupole moment tensor defined as

$$
I^{i j}(t)=\int d^{3} y y^{i} y^{j} T^{00}
$$

For the modified equation with $b^{\mu}=\left(b^{0}, \mathbf{0}\right)$ and since the bumblebee field does not couples with the matter fields, we may that $J_{\mu \nu}=16 \pi G T_{\mu \nu}$. Therefore the solution for the perturbation is

$$
h_{i j}(t, \mathbf{r})=\frac{2 G}{r} \frac{d^{2} I_{i j}}{d t^{2}}\left(t_{r}^{\prime}\right),
$$


where $t_{r}^{\prime}=x^{0}-r \sqrt{1+\xi\left(b^{0}\right)^{2}}$ is the modified retarded time. Therefore, all the parameters of the wave, besides the slower propagation velocity, must be the same.

For the $b^{\mu}=(0, \mathbf{b})$ configuration, the solution is

$$
h_{i j}(t, \mathbf{r})=\frac{2 G}{r}\left[\left(1-\frac{\xi b^{2}}{2} \cos 2 \theta_{b}\right) \frac{d^{2} I_{i j}}{d t^{2}}\left(t_{r}\right)+\frac{\xi(\mathbf{b} \cdot \mathbf{r})^{2}}{2 r} \frac{d^{3} I_{i j}}{d t^{3}}\left(t_{r}\right)\right] .
$$

In this equation, we can observe once more the existence of anisotropy, the amplitude of the wave is modified, therefore, the existence of the bumblebee field can be attested in a experiment. Since the quadrupole moment remained unmodified, the frequency of the wave must not change. Other peculiarity is the third derivative of $I_{i j}$ that appeared as consequence of the expansion of the Green function to the second order.

\section{Conclusion}

In this work, we searched the effects of the spontaneous LSB on the production of the GW. We introduced the LSB by the coupling of the graviton with a vector $B^{\mu}$ known as bumblebee vector field that has a nonzero VEV. This leads to a modified wave equation for the graviton propagation.

Analyzing this new relation we determine the solution for this model in two special configurations. In the first one, we considered a timelike configuration for $b^{\mu}$, in this case, only the the velocity of propagation of the wave were smaller by a factor of $\sqrt{1+\xi\left(b^{0}\right)^{2}}$. In the second case, a spacelike configuration was considered for the bumblebee VEV. The solution showed the existence of anisotropy in the solution, which is well known in the literature [8], modifications in the amplitude and presence of a third derivative of the quadrupole moment.

\section{References}

[1] V. A. Kosteleckỳ and S. Samuel, Phys. Rev. D 39, n. 2, p. 683, 1989

[2] R. Bluhm and V. A. Kostelecký, Phys. Rev. D 71, 065008 (2005).

[3] R. Bluhm, S-H. Fung, and V. A. Kostelecký, Phys. Rev. D 77, 065020 (2008).

[4] C. A. Hernaski, Phys. Rev. D 90, 124036 (2014).

[5] R. V. Maluf, et al, Physical Review D, v. 88, n. 2, p. 025005, 2013.

[6] R. V. Maluf, et al, Physical Review D, v. 90, n. 2, p. 025007, 2014.

[7] Q. G. Bailey, V. A. Kostelecký, Physical Review D, v. 74, n. 4, p. 045001, 2006.

[8] V. A. Kostelecký, and M. Matthew, Physical Review D 66.5 (2002): 056005. 